\title{
Application of DIC to monitor reinforced concrete structures
}

\section{Luis Saucedo-Mora ${ }^{1}, \mathrm{M}^{\mathrm{a}}$ Carmen Andrade Perdrix, Cecilio López Hombrados, Javier Barroso and Ana Zamora Bragado}

\author{
DOI: https://doi.org/10.5592/CO/BSHM2017.4.3 \\ Institute Eduardo Torroja for Construction Sciences (CSIC), Madrid, Spain
}

\section{E-mails: luis.saucedomora@ietcc.csic.es ${ }^{l}$}

\begin{abstract}
The reinforced concrete structures need to be monitored to ensure their structural integrity, but sometimes those measurements are very local and the instrument is complex to locate physically in the structure and may interfere on it. Digital Image Correlation, DIC, is a non-contact and non-destructive experimental technique capable to measure the displacement field in a big region of a structure with a great accuracy. This allows extracting valuable information from the fracture processes of reinforced concrete structures. Critical for the evaluation of the structural integrity. The identification of the energy dissipated by the structure is essential for the identification of the strength mechanisms that are failing in the structure, and to identify a proper repair. In this paper the penetration of a prestress rebar in concrete is measured with this technique and the energy dissipated by different fractures is fully observed. Comparison is made with traditional measurement techniques. Also, using Fracture Mechanics other valuable information is extracted from the fracture processes of the reinforced concrete beam, such as the Mode I and Mixed Mode fracture energy released at each loading step, which is essential to evaluate the elastic energy that the structure can accumulate before collapse. The examples enable to anticipate the importance of DIC for future studies at large scale of fracture in concrete and other materials related to construction.
\end{abstract}

Keywords: Digital Image Correlation, Fracture Mechanics, damage

\section{Introduction}

Digital Image Correlation (DIC) is a robust, non-destructive and non-contact experimental technique widely used in the last decades for micromechanical characterization of materials (Chu et al 1985). It uses images of a surface to measure its local displacements, being the input of DIC a pair of images (i.e. the reference and deformed), which are divided in windows to correlate them and measure its displacements (Pan et al 2009). The use of this technique was extended, although still is very scarcely employed, to concrete structures in the last years with successful results. The great complexity and size of the concrete elements were a drawback for the use of DIC, but the information that can be extracted with it is essential for the full characterization of the fracture processes of concrete, which has encouraged its application. Thus, among others, in 2006 Küntz et al. applied DIC to measure the stability of a shear crack in a reinforced concrete bridge during loading. In 2012 Lee et al. compared the measurements done with DIC in a reinforced concrete structure with foil and vibrating wire strain gauges. They concluded that DIC is more versatile and as accurate as the others. In 2013 Dutton et al. used DIC to measure the curvature of a reinforced concrete beam of $3.8 \mathrm{~m}$ long. They did it imaging 2 regions of $600 \times 400 \mathrm{~mm}$ were the deflection was calculated. And in 2014 Fayyad and Lees used it to measure the opening of a crack in a notched beam of $0.8 \mathrm{~m}$ long.

This paper will focus on exploring the possibilities of application of DIC as a health monitoring technique for civil structures, mainly motivated by its not contacting and non-destructive nature. The use of DIC to characterize the fracture of a reinforced concrete beam is also very useful to understand the complex fracture processes (Bazant and Planas 1997) and the evolution of the crack behaviour. Its combination with fracture mechanics allows us to extract more characteristics of the fracture processes such as the relationship between Mode I and Mode II and the transition between both. For this the stress intensity factors are used and calculated from the strain field ahead of the crack tip. There are many solutions for this calculation such as the interaction integral technique (Dolbow et al 2002) or the method of Yoneyama el at. In 2007, but in this paper we are using the classical polar equations of the linear elastic fracture mechanics (Shah et al 1995). In this paper we are able to measure the discontinuity created by a crack, the crack opening along the height of the beam and to characterize a Mode I and a Mixed-Mode fracture. All those aspects, with the Fracture Process Zone (SaucedoMora et al 2012), are the keys to characterize the integrity of the reinforced concrete structures. 


\section{Experimental: Fracture test of a prestress beam}

In this case the DIC method was applied to a three point bending test in a concrete beam with 4 prestressed rebars and a span of $700 \mathrm{~mm}$ and a $250 \times 200 \mathrm{~mm}$ section. The concrete has a compressive strength of $75 \mathrm{MPa}$, and the beam has 4 prestressed longitudinal rebars of a 1670C steel with a diameter of $7.5 \mathrm{~mm}$ located 2 at $50 \mathrm{~mm}$ from the top of the beam, and other 2 at $40 \mathrm{~mm}$ from the bottom. Without any shear reinforcement. The imaged area was $390 \times 260 \mathrm{~mm}$ located beneath the loading point in the centre of the beam front. The images were taken with a camera Nikon D7200, with a size of 6000x4000 pixels (i.e. 24 Megapixels). Resulting in a pixel size of $0.065 \mathrm{~mm}$. The measurement has an error of $5 \mu \mathrm{m}$, measured with an undeformed image displaced with a Rigid Body Movement. After the calculation with the deformed images, the displacement field was corrected for Rigid Body Movements, rotation and out of plane movements. Figure 1 is an example of a DIC treatment of an image at an early stage of the cracking process (i.e. half of the peak load), it shows the relative strain between the loaded and the unloaded beam, without considering its initial prestress strain. At the bottom the crack initiation can be identified as a hot spot in Figure 1a. Also, as expected, there are compressive strains in the top part of the beam. Figure 1b shows the deflection of the beam, measured through DIC at the bottom of the image, and it's comparison with the analytical deformation given by the beam theory. In this case any big discontinuity in the deflection can be clearly identified, as in Figure 4a for higher loads.

a)

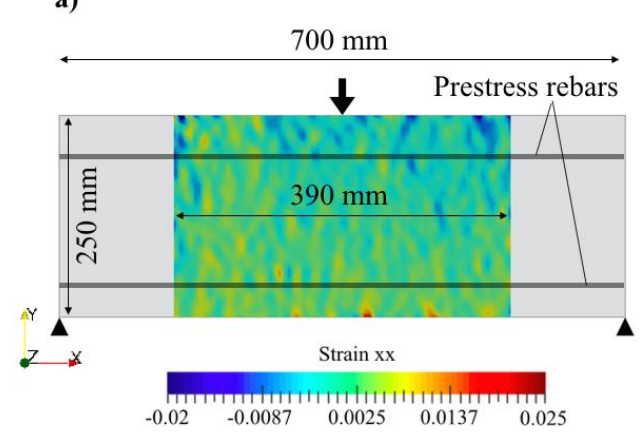

b)

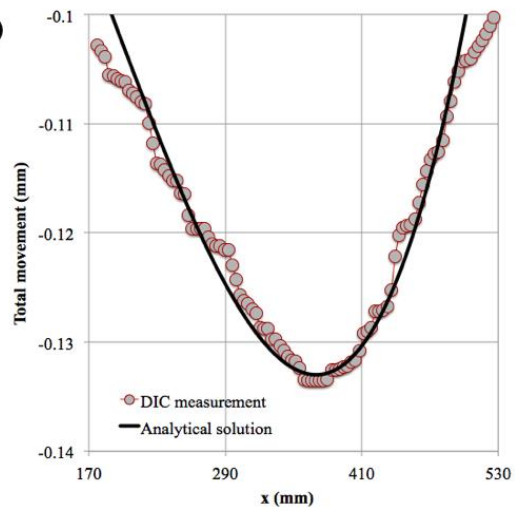

Fig. 1. DIC of the beam at $220 \mathrm{kN}$ (45\% of the peak load); a) geometry of the beam and region imaged with the strain in $\mathrm{x}$ and b) deflection of the beam measured with DIC compared with the analytical solution.

\section{Results}

The beam was tested with load/unload cycles with increasing peaks. For higher loads (Figure 2), the crack grows from the location spotted in Figure 1a at an earlier stage with the DIC analysis. Because the displacement field of the imaged region of the beam is extracted with DIC, this displacement can be magnified (100 times in) to plot the deformed shape of the beam with the strain field superimposed, as in Figure 2. This amplification shows that the displacements measured are not noisy, producing a homogeneous deformation. Also, applying an arbitrary threshold of 0.1 in the strain, the crack can be identified. Figure 2 shows the strain concentration at the crack tip and the Fracture Process Zone, as well as the regions under compression on the top of the beam.

Figure 2 shows the interaction between the Mode I crack originated beneath the loading point, and the compressive region on the top of the beam. As the crack grows from $280 \mathrm{kN}$ to $320 \mathrm{kN}$ the compressed region is displaced from the centre of the beam, growing the crack around this region at $360 \mathrm{kN}$. In Figure 2 the regions in purple are confined under compression and their displacement to one side of the loading point creates nonsymmetry in the curvature of the beam. Also the displacement of the compressive regions leads to an increment of the shear in the right side of the beam that ends with a shear crack, which will be later introduced.

This analysis allows us to characterize in detail the crack. Figure $3 \mathrm{a}$ shows an analysis of the orientation of the maximum principal strain in a $100 \times 100 \mathrm{~mm}$ region of the crack zone at $300 \mathrm{kN}$. Here, because the eigenvectors are scaled with the value of their associated eigenvalue, we can see how the orientation of those vectors defines the fracture path. As well, it can be identified how the strain of the crack decreases as it approaches the crack tip. In the rest of the sample the eigenvectors of the principal maximum strains with low values are not oriented in any preferably direction. It shows the complex elastic deformation of concrete due to the different local stiffness of the aggregates and matrix that conform the microstructure. 

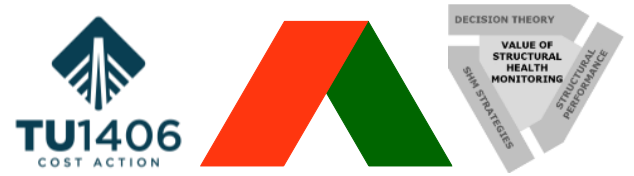

a)

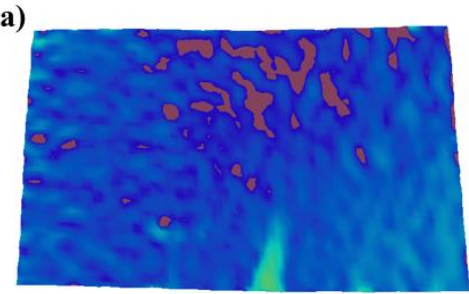

c)

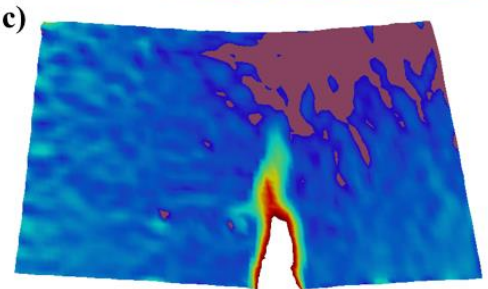

b)

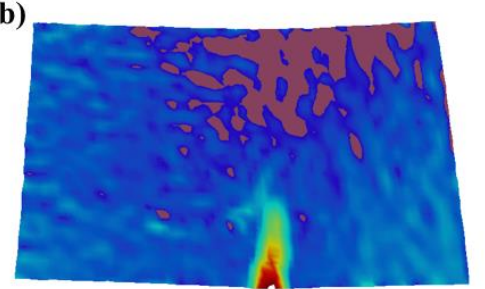

d)

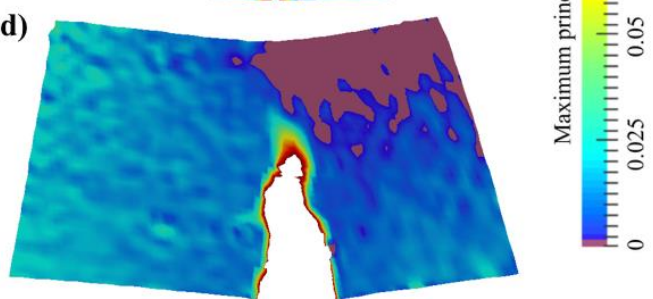

$20 \mathrm{~mm}$

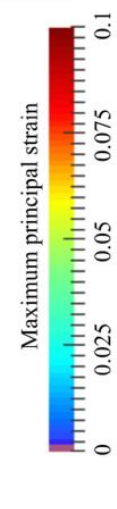

Fig. 2. Deformed shape magnified 100 times and superimposed strain field of the beam loaded at: a) $260 \mathrm{kN}$, b) $280 \mathrm{kN}$, c) $320 \mathrm{kN}$ and d) $360 \mathrm{kN}$.

The regions with higher strains of Figure $3 \mathrm{a}$ are not the real strains of the material and are just the derivative of the jump in the $\mathrm{x}$ displacement between the crack faces. Only in the elastic regions those strains are the real strains of the material. Extracting the jump between the crack faces along the beam height for different loading steps we can measure the crack opening shown in Figure 3b. In this figure the crack tip was identified as a kink in the small linear elastic deformation that generates the variation of the crack opening plotted in the figure. As the load increases, the tip (in Figure $3 \mathrm{~b}$ only the open crack is plotted) moves closer to the top of the beam, and the slope of the opening becomes less inclined.
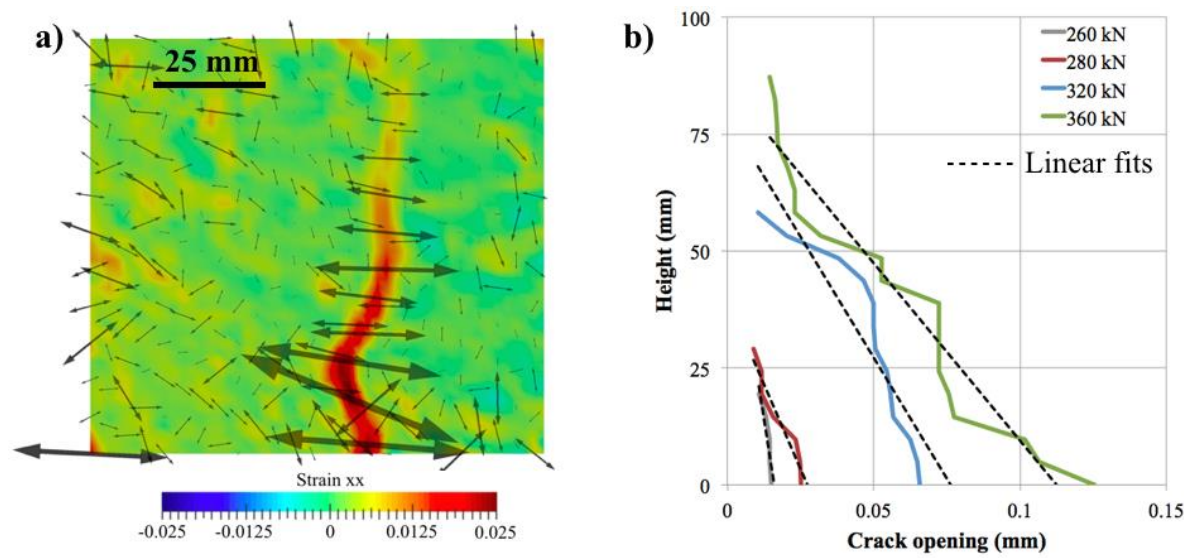

Fig. 3. Analysis of a region of interest of $100 \times 100 \mathrm{~mm}$ around the crack: a) strains field in $\mathrm{x}$ and eigenvectors of the maximum principal strain and b) crack opening versus the vertical position in the beam.

The crack opening of Figure $3 \mathrm{~b}$ is just the jump of the total displacement along the beam. Figure 4a shows the total displacement measured at the bottom of the beam at $260 \mathrm{kN}, 280 \mathrm{kN}, 320 \mathrm{kN}$ and $360 \mathrm{kN}$, close to the final load of $490 \mathrm{kN}$. Figure $4 \mathrm{a}$ is the evolution of Figure $2 \mathrm{~b}$, remarking the effect of the damage. The main difference introduced by the crack is that in Figure $3 \mathrm{~b}$ almost all the displacement is given by the $y$ deflection, and in Figure 4a the jump between the crack faces, mainly in $x$ for a Mode I crack, is governing the total movement along the bottom of the beam. Measurements at each load/unload cycle were done with a gauge of $80 \mathrm{~mm}$ at the left and right of the Mode I crack, showing in Figure $4 \mathrm{~b}$ the evolution of the residual strains in the beam. This result shows that apart from the main crack, the damage is spread by the rebar around the main crack region. This is a direct effect of the pull out of the rebar from the crack faces and the transfer of stresses between them, which doesn't relax the regions around the crack faces as in the materials without any kind of reinforcement, prestress rebars in this case. The loaded results of Figure $4 \mathrm{~b}$ are a combination of the elastic deformation and the opening of the microcracks shown during the unload. 

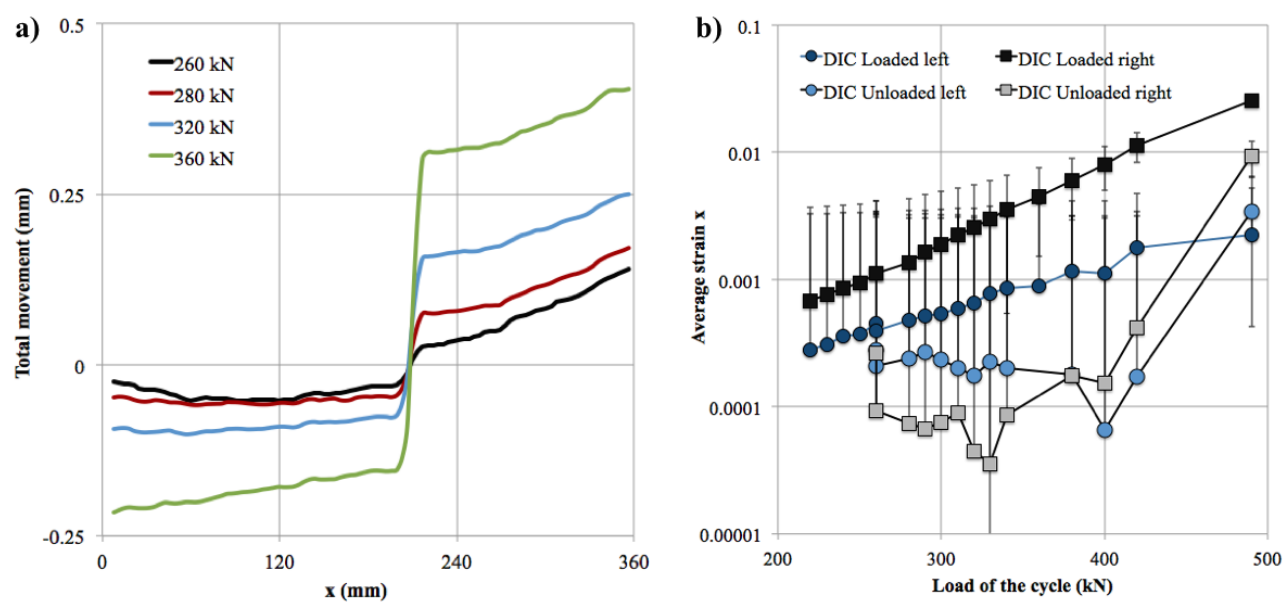

Fig. 4. a) movement profile along the bottom of the beam for different loads and b) measurement of the average strain on the left and right of the crack with a gauge of $80 \mathrm{~mm}$ for the beam loaded and unloaded.

$70 \mathrm{kN}$ before the final load, at $420 \mathrm{kN}$, another crack appeared on the right of the Mode I crack previously described. This crack goes from the region with high shear strain at the bottom of the beam to the region on the top confined under compression (Figure 2d). This crack grows dynamically, appearing with its $85 \mathrm{~mm}$ length between $400 \mathrm{kN}$ and $420 \mathrm{kN}$. As a comparison, the Mode I crack of the center grows $20.1 \mathrm{~mm}$ in this gap of 20 $\mathrm{kN}$.

\section{Conclusions}

The paper presents the potential applications of the Digital Image Correlation Technique to monitor structural concrete, paving the way for future developments and applications in civil structures. Complex mechanisms that involve damage and the lost of capabilities of the structural element are measured successfully using this technique, with a simple setup. The novelty of the methodology described in this paper is the capability to describe not only the crack location and opening, also the way that the elastic energy is released at each fracture step. Also the non-contact and non-destructive nature of the technique enhances its applicability to structures in extreme environments and locations complex to reach, showing the potentiality of this technique to be used for structural health monitoring.

\section{Acknowledgements}

The authors want to acknowledge Rogelio Sánchez Verdasdo for his support during the test with the manipulation and setup of the cameras. Luis Saucedo-Mora wants to acknowledge the financial support given by the Secretariat of State for Research, Development and Innovation of the Spanish government under the grant IJCI-2014-19362 associated to a Juan de la Cierva Incorporation Fellowship.

\section{References}

T. C. Chu, W. F. Ranson, M. A. Sutton. Applications of digital-image-correlation techniques to experimental mechanics. Experimental Mechanics. 1985. 25(3): pp. 232-244. doi: 10.1007/BF02325092

B. Pan, K. Qian, H. Xie and A. Asundi. Two-dimensional digital image correlation for in-plane displacement and strain measurement: a review. Measurement Science and Technology. 2009. 20: 062001. doi: http://dx.doi.org/10.1088/0957-0233/20/6/062001

M. Küntz, M. Jolin, J. Bastien, F. Perez, F. Hild. Digital image correlation analysis of crack behavior in a reinforced concrete beam during a load test. Canadian Journal of Civil Engineering, 2006, 33:1418-1425, doi: $10.1139 / 106-106$

C. Lee, W.A. Take, N. A. Hoult, M. ASCE. Optimum Accuracy of Two-Dimensional Strain Measurements Using Digital Image Correlation. Journal of Computing in Civil Engineering, 2012, 26(6): 795-803. doi: 10.1061/(ASCE)CP.1943-5487.0000182

M. Dutton, W. Take, N. Hoult. Curvature Monitoring of Beams Using Digital Image Correlation. Journal of Bridge Engineering, 2014, 19(3). doi: 10.1061/(ASCE)BE.1943-5592.0000538, 05013001. 

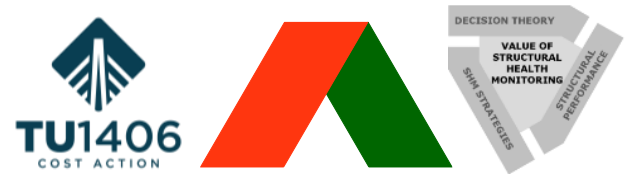

The Value of Structural Health Monitoring for the reliable Bridge Management

T.M. Fayyad, J.M. Lees. Application of Digital Image Correlation to reinforced concrete fracture. Procedia Materials Science, 2014, 3: 1585 - 1590. doi: 10.1016/j.mspro.2014.06.256

Z.P. Bazant, J. Planas. Fracture and Size Effect in Concrete and Other Quasibrittle Materials. CRC Press, 1997. ISBN: 084938284X, 9780849382840

S. Yoneyama, T. Ogawa, and Y. Kobayashi, Evaluating Mixed-Mode Stress Intensity Factors from Full-Field Displacement Fields Obtained by Optical Methods. Engineering Fracture Mechanics, 2007, p. 1399-1412.

J. Dolbow, M. Gosz. On the computation of mixed-mode stress intensity factors in functionally graded materials. International Journal of Solids and Strucures, 2002, 39(9): 2557-2574. doi:10.1016/S00207683(02)00114-2

S.P. Shah, S.E. Swartz, C. Ouyang. Fracture Mechanics of Concrete: Applications of Fracture Mechanics to Concrete, Rock and Other Quasi-Brittle Materials. Wiley, 1995, ISBM: 978-0-471-30311-4

L. Saucedo-Mora, R.C. Yu, G. Ruiz. Fully-developed FPZ length in quasi-brittle materials. International Journal of Fracture. 2012, 178:97-112. doi: 10.1007/s10704-012-9769-0 